[0212-7199 (2005) 22: 7; pp 317-322] ANALES DE MEDICINA INTERNA Copyright (C) 2005 ARAN EDICIONES, S.L

AN. MED. INTERNA (Madrid) Vol. 22, N. $^{\circ} 7$, pp. $317-322,2005$

\title{
Remodelación esplácnica secundaria a hipertensión portal prehepática experimental
}

\author{
M. T. CORCUERA PINDADO, M. P. NAVA HIDALGO ${ }^{1}$, A. ANGULO BURGOS, \\ M. A. ALLER REYERO ${ }^{2}$, F. GÓMEZ AGUADO, I. CASADO FARIÑAS, \\ M. J. ALONSO MARTÍN, A. JAIME PÉREZ² \\ Servicio de Anatomía Patológica. Hospital Carlos III. 'Departamento de Fisiología \\ (Fisiología II). Facultad de Biológicas. Universidad Complutense. Madrid. ${ }^{2}$ Cátedra de \\ Cirugía. Departamento de Cirugía I. Facultad de Medicina. Universidad Complutense. \\ Madrid
}

SPLANCHNIC REMODELING SECONDARY TO EXPERIMENTAL PREHEPATIC PORTAL HYPERTENSION

\section{RESUMEN}

Objetivo: La hipertensión portal, en tanto que inductora de una respuesta inflamatoria intestinal, causaría a largo plazo remodelación epitelial y vascular esplácnicas. Con el objeto de verificar esta hipótesis se ha realizado éste trabajo experimental.

Método: Se han estudiado como alteraciones estructurales propias de remodelación epitelial intestinal y vascular mesentérica la densidad de células caliciformes y el diámetro de las ramas de la vena mesentérica, respectivamente, en ratas con ligadura parcial de la vena porta a corto (1 mes) y largo (1 año) plazo.

Resultados: La hiperplasia de células caliciformes en intestino delgado es máxima al año de evolución de la hipertensión portal y se asocia con dilatación de las ramas distales $\left(3^{\text {er }}\right.$ y $4^{\circ}$ orden) de la vena mesentérica superior.

Conclusión: La remodelación esplácnica que ocurre a largo plazo en la hipertensión portal experimental sugiere la existencia de un proceso inflamatorio crónico en ésta patología.

PALABRAS CLAVE: Hipertensión portal. Inflamación. Remodelación. Rata.

\begin{abstract}
Objective: Portal hypertension as an inducer of intestinal inflammatory response would cause epithelial and splanchnic vascular remodeling in the long-term. This experimental study was carried out to verify this hypothesis.

Method: Structural alterations characteristic of intestinal epithelial and mesenteric vascular remodeling, the density of goblet cells and the diameter of mesenteric vein branches were studied, respectively, in rats with partial portal vein ligation in the short (1 month) and long-term (1 year).

Results: Hyperplasia of goblet cells in the small intestine (duodenum, jejunum, ileum) is maximum after 1 year of evolution of the portal hypertension and is associated with dilatation of the distal branches (3rd and 4 th order) of the superior mesenteric vein.

Conclusion: Long-term splanchnic remodeling in experimental portal hypertension suggests the existence of a chronic inflammatory process in this clinical condition.
\end{abstract}

KEY WORDS: Portal hypertension. Inflammation. Remodeling. Rat.

Corcuera Pindado MT, Nava Hidalgo MP, Angulo Burgos A, Aller Reyero MA, Gómez Aguado F, Casado Fariñas I, Alonso Martín MJ, Jaime Pérez. A. Remodelación esplácnica secundaria a hipertensión portal prehepática experimental. An Med Interna (Madrid) 2005; 22: 317-322.

\section{INTRODUCCIÓN}

Se denomina "enteropatia hipertensiva portal" a las alteraciones del tracto gastrointestinal secundarias a la hipertensión portal (1). Ya que las alteraciones estructurales básicas en el tracto gastrointestinal, son vasculares, con aumento del tama-

Trabajo presentado parcialmente en el VI Congreso Virtual Hispanoaméricano de Anatomía Patológica (http:lconganat.sld.cu/6CVHAP).

*Trabajo realizado en parte con una Ayuda para Proyectos de Investigación. Consejería de Sanidad. Junta de Comunidades de Castilla La Mancha (ref. $\mathrm{n}^{\circ}$ 01011). ño y número de los vasos, también se han propuesto las denominaciones de "gastroenteropatía congestiva" y "vasculopatía intestinal hipertensiva portal" (1-3).

En ratas con hipertensión portal prehepática, por ligadura estenosante de la vena porta, también se ha demostrado a las seis semanas de evolución el aumento del número y diámetro de los vasos submucosos duodenales (4). Sin embargo la coexistencia de un significativo aumento de células cebadas, permite sugerir que tanto la hipertensión portal como dichas células inflamatorias podrían estar implicadas en la producción de la vasculopatía intestinal hipertensiva portal (4).

La supuesta naturaleza inflamatoria de la enteropatía hipertensiva portal, es una atractiva hipótesis porque en este caso tanto las alteraciones precoces como tardías podrían ser

Trabajo aceptado: 31 de marzo de 2005 
atribuidas a dicha causa. En particular los cambios estructurales que se producen a largo plazo constituirían los propios de un proceso de remodelación, similar al descrito en otros procesos inflamatorios crónicos (5).

Para demostrar las alteraciones estructurales que se producen en la hipertensión portal prehepática experimental como resultado de un proceso inflamatorio, se realizó un estudio cuantitativo de las ramas de la vena mesenterica superior y de las células caliciformes en el intestino delgado, en tanto que elementos que reflejan la remodelación esplácnica macrovascular y epitelial, respectivamente.

\section{MATERIAL Y MÉTODO}

Se utilizaron ratas macho de la cepa Wistar procedentes del Animalario de la Universidad Complutense de Madrid, con pesos corporales que oscilaron entre 226 y $305 \mathrm{~g}$.

El procedimiento experimental empleado en este estudio se efectuó según los principios para el cuidado y la utilización de los animales de laboratorio publicados en España en el R.D. 223/1988.

\section{DISEÑO EXPERIMENTAL}

Para su estudio los animales fueron divididos en cuatro grupos: grupos controles de un mes (grupo I, $\mathrm{n}=11$ ) y un año (grupo III, $n=13$ ) de evolución en los cuales los animales no sufrieron ningún tipo de intervención. Grupos con hipertensión portal prehepática por triple ligadura estenosante de la vena porta (TEVP), de un mes (grupo II, $\mathrm{n}=21$ ) y de un año (grupo IV, $\mathrm{n}=17$ ) de evolución postoperatoria. Todos los animales fueron sacrificados por sobredosis de eter y se determinaron los pesos corporal (PC), hepático $(\mathrm{PH})$, esplénico (PE) y testicular (PT).

\section{TÉCNICA QUIRÚRGICA DE HIPERTENSIÓN PORTAL}

Las ratas se anestesian con ketamina $(80 \mathrm{mg} / \mathrm{kg})$ y xilacina $(12 \mathrm{mg} / \mathrm{kg}$ ) por vía intramuscular. Se realiza una laparotomia media y se desplazan las asas intestinales y el estómago a la izquierda del animal. Los lóbulos hepáticos medio y lateral izquierdo se desplazan en sentido craneal y se mantienen en esta posición mediante una torunda de algodón subdiafragmática. A continuación se diseca la vena porta en toda su longitud y la hipertensión portal se realiza por triple ligadura estenosante de la vena porta (TEVP). Se realizan tres ligaduras estenosantes en las porciones superior, media e inferior de la vena porta, respectivamente, las cuales son mantenidas en posición por la fijación previa de las ligaduras a una guía de silastic (6). Las estenosis son calibradas por ligadura simultánea (seda de 4/0) alrededor de la vena porta y de una aguja de $20 \mathrm{G}$, que después se retira (7). La laparotomia se cierra en dos planos con catgut y seda de $2 / 0$.

\section{DETERMINACIÓN DE LA PRESIÓN VENOSA PORTAL}

La presión portal se mide por una técnica indirecta de punción intraesplénica (8) insertando una aguja 20G en el parén- quima esplénico que se conecta a un catéter PE-50. Este catéter, a su vez, se conecta a un registrador de presión Powerlab 200 (ML 201) y a un transductor de presión Sensonor (SN844), asociados a un programa de ordenador Chart V 4.0 (ADI Instruments). El sistema se calibra antes de cada experimento y el punto de referencia 0 se colocó en el punto medio del animal, $1 \mathrm{~cm}$ por encima de la mesa de operaciones. Estudios previos han demostrado una excelente correlación entre la medición indirecta de la presión portal por punción intraesplénica y la medición directa de la presión portal por canulación de la vena mesentérica superior (9).

\section{MÉTODO DE ESTUDIO DE LA CIRCULACIÓN COLATERAL}

Se estudiaron las áreas en las que se desarrolla la circulación venosa colateral en los animales con hipertensión portal (Grupos II y IV): esplenorenal (colaterales esplenorenales superior e inferior), gastroesofágica (colaterales paraesofágicas), colorectal (colateral pararectal) e hilio hepático (portoportal y vena hepática accesoria) (10).

\section{ESTUDIO DE LA VASCULOPATÍA VENOSA MESENTÉRICA}

Se ha denominado vasculopatía venosa mesentérica a la dilatación y tortuosidad de las ramas de la vena mesentérica superior. Se han considerado tres grados de vasculopatía venosa mesentérica: grado 0: aspecto macroscópico normal de las ramas de la vena mesentérica superior; grado I: dilatación y tortuosidad de las mencionadas ramas, secundaria a la maniobra de Pringle; y grado II: la dilatación y tortuosidad de las ramas de la vena mesenterica superior son espontáneas (11).

La vasculopatía venosa mesentérica se estudió principalmente en el mesenterio de la región ileocecal, donde se visualizan con facilidad las ramas de la vena mesentérica superior. Esto es, las ramas de primer y segundo orden ó proximales por su vecindad con la vena mesentérica superior, y las ramas de tercer y cuarto orden o distales por su vecindad con el intestino (íleon y ciego) (8). El estudio cuantitativo de la vasculopatía venosa mesentérica se efectuó a partir de imágenes digitalizadas del mesenterio mediante un sistema de análisis de imagen basado en el software QWin (Leica Microsystems). Una vez calibrado el sistema, se midió el diámetro de la vena mesentérica superior, de sus ramas proximales y distales, asi como de la vena mesentérica inferior en su trayecto pararectal.

\section{ESTUDIO HISTOLÓGICO INTESTINAL}

En todos los animales se resecó la segunda porción del duodeno y segmentos de yeyuno (a $10 \mathrm{~cm}$ del angulo de Treitz) e ileon (a $3 \mathrm{~cm}$ de la válvula ileocecal) que fueron perfundidos intraluminalmente con suero salino. Posteriormente las muestras fueron fijadas con formol $10 \%$, deshidratadas e incluidas en parafina. Secciones de 5 ?m perpendiculares a la luz intestinal fueron teñidas con hematoxilina-eosina, visualizadas con un microscopio digital Leica DM 5000B y las imágenes obtenidas fueron valoradas con un sistema de análisis de imagen (QWin, Leica Microsystems). En todas las mues- 
tras se cuantificó el número de células caliciformes en un área de al menos $150.000 \mu^{2}$ y el resultado se expresó como número de células caliciformes $/ 1.000 \mu^{2}$ de epitelio glandular. Asimismo fue cuantificado el número de células en mitosis en las criptas duodenales, yeyunales e ileales.

\section{ANÁLISIS ESTADÍSTICO}

Las variables se expresan como media \pm desviación estándar. El test de la t de Student para datos independientes fue utilizado para realizar el contraste de hipótesis. entre cada grupo de ratas con hipertensión portal en relación con su respectivo grupo control Las pruebas de ANOVA y NewmanKeuls fueron utilizadas para la comparación de las variables entre los grupos I, II, III y IV. Los resultados se consideran estadísticamente significativos si $\mathrm{p}<0,05$.

\section{RESULTADOS}

\section{PESO CORPORAL Y DE LOS ÓRGANOS}

Los animales del grupo IV (TEVP-1 año) presentan una disminución del peso corporal que es estadísticamente significativa $(\mathrm{p}<0,01)$ respecto del grupo III (control 1 año) (Tabla I). Respecto del peso hepático, la disminución en los animales con TEVP es más intensa en el grupo II (TEVP-1 mes) que en el grupo IV (TEVP-1 año) (Tabla I).

La esplenomegalia es común a ambos grupos de animales con TEVP, si bien, el incremento del peso esplenico en el grupo IV (TEVP-1 año) es superior respecto del grupo II (TEVP1 mes) (Tabla I).

El peso testicular en el grupo II (TEVP-1 mes) presenta una disminución estaditicamente significativa $(\mathrm{p}<0,05)$ respecto del grupo I (control 1 mes) (Tabla I).

\section{PRESIÓN PORTAL}

En todos los animales con hipertensión portal se produce un incremento de la presión portal respecto de los animales control que es mayor al mes $(\mathrm{p}<0,001)$ que al año de evolución $(\mathrm{p}<0.05)$ (Tabla I).

\section{CIRCULACIÓN COLATERAL}

Todos los animales con TEVP presentan circulación colateral portosistemica. En el grupo II predominan los tipos esplenorrenal $(90,9 \% ; n=10)$, pararrectal $(81,81 \% ; n=9)$ y paraesofágica $(72.72 \% ; n=8)$. Los animales del grupo IV presentan colaterales esplenorrenales $(66,66 \% ; n=4)$ y pararrectales $(66,66 \% ; n=4)$, pero disminuyen las colaterales paraesofágicas $(33,33 \% ; n=2)$. Asimismo, en el 83,33\% de los animales $(n=5)$ existe circulación colateral portohepática a traves de la vena hepática accesoria.

\section{VASCULOPATÍA VENOSA MESENTÉRICA}

Todos los animales con TEVP de 1 mes de evolución (grupo II) presentan vasculopatía venosa mesenterica ya de grado I $(36,36 \% ; n=4 ;)$ o de grado II $(63,64 \% ; n=7)$. Sin embargo en los animales con TEVP de 1 año de evolución (grupo IV) la vasculopatía venosa mesenterica no es generalizada ya que no se presenta en el $16,66 \% \%(n=1)$ de los casos. A su vez es más frecuente la vasculopatía mesentérica de grado I $(60 \% ; n=6)$ que la de grado II $(40 \% ; n=2)$.

En las ratas con hipertensión portal de 1 mes de evolución (grupo II), se produce un incremento del diámetro de la vena mesenterica $(\mathrm{p}<0,05)$, así como de sus ramas de primer y segundo orden (proximales) $(\mathrm{p}<0,05)$, respecto de las ratas control (grupo I) (Tabla II).

Por el contrario los animales con hipertensión portal de 1 año de evolución postoperatoria (grupo IV) presentan un incremento del diámetro de las ramas de la vena mesentérica de tercer y cuarto orden (distales) $(\mathrm{p}<0,05)$, respecto de los animales control (grupo III) (Tabla II y Fig. 1).

En los animales del grupo II (TEVP 1 mes) se produce un gran incremento del diámetro de la vena hemorroidal (que se continua con la vena mesentérica inferior) respecto de su control (grupo I). A su vez en los animales del grupo IV (TEVP 1 año) el diámetro de la vena hemorroidal es similar al que presentan los animales control (grupo III) (Tabla II).

\section{TABLA I}

INCREMENTO DE PESO CORPORAL ( $\triangle \mathrm{PC}$ ), PESO HEPÁTICO (PH), PESO ESPLÉNICO (PE), PESO TESTICULAR (PT) Y PRESIÓN PORTAL (PP) EN LOS ANIMALES DEL GRUPO I (CONTROL) Y DEL GRUPO II (CON TRIPLE LIGADURA DE LA VENA PORTA) AL MES DE EVOLUCIÓN Y EN LOS GRUPOS III (CONTROL) Y IV (CON TRIPLE LIGADURA DE LA VENA PORTA) AL AÑO DE EVOLUCIÓN

\begin{tabular}{lccccc}
\hline & $\begin{array}{c}\Delta P C \\
(g)\end{array}$ & $\begin{array}{c}P H \\
(g)\end{array}$ & $\begin{array}{c}P E \\
(g)\end{array}$ & $\begin{array}{c}P T \\
(g)\end{array}$ & $\begin{array}{c}P P \\
(\mathrm{mmHg})\end{array}$ \\
\hline Grupo I $(\mathrm{n}=11)$ & $64,72 \pm 29,3$ & $12,01 \pm 2,64$ & $0,49 \pm 0,06$ & $3,44 \pm 0,29$ & $7,16 \pm 0,95$ \\
Grupo II $(\mathrm{n}=21)$ & $82,49 \pm 29,08$ & $9,98 \pm 1,55$ & $1,00 \pm 0,16 * *$ & $2,87 \pm 0,57^{*}$ & $14,28 \pm 3,16 * *$ \\
Grupo III $(\mathrm{n}=13)$ & $361,02 \pm 58,32$ & $16,76 \pm 2,87$ & $0,91 \pm 0,10$ & $3,65 \pm 0,38$ & $6,72 \pm 1,52$ \\
Grupo IV $(\mathrm{n}=17)$ & $268,02 \pm 78,03 * *$ & $15,84 \pm 7,02$ & $1,19 \pm 0,19 * *$ & $3,51 \pm 0,51$ & $9,05 \pm 2,84 *$ \\
\hline
\end{tabular}

$\mathrm{x} \pm \mathrm{DE} ;{ }^{*} \mathrm{p}<0,05 ;{ }^{*} \mathrm{p}<0,01 ;{ }^{* *} \mathrm{p}<0,001$ : Valores estadísticamente significativos respecto de su valor control. 
TABLA II

DIÁMETRO DE LA VENA MESENTÉRICA (VM), DE LAS VENAS MESENTÉRICAS DE PRIMER, SEGUNDO, TERCER Y CUARTO ORDEN Y DE LA VENA HEMORROIDAL (VH) EN LOS ANIMALES DE LOS GRUPOS I (CONTROL) Y II ( CON TRIPLE LIGADURA ESTENOSANTE DE LA VENA PORTA) AL MES DE EVOLUCIÓN Y EN LOS GRUPOS III ( CONTROL) Y IV (CON TRIPLE LIGADURA ESTENOSANTE DE LA VENA PORTA) AL AÑO DE EVOLUCIÓN

\begin{tabular}{|c|c|c|c|c|c|c|}
\hline & $\begin{array}{l}V M \\
(\mu \mathrm{m})\end{array}$ & $\begin{array}{c}\text { VM } 1^{\text {er }} \text { orden } \\
(\mu \mathrm{m})\end{array}$ & $\begin{array}{c}\mathrm{VM} 2^{\circ} \text { orden } \\
(\mu \mathrm{m})\end{array}$ & $\begin{array}{c}\text { VM } 3^{\text {er }} \text { orden } \\
(\mu \mathrm{m})\end{array}$ & $\begin{array}{l}\text { VM } 4^{\circ} \text { orden } \\
(\mu \mathrm{m})\end{array}$ & $\begin{array}{c}V H \\
(\mu m)\end{array}$ \\
\hline Grupo I $(n=6)$ & $784,70 \pm 124,91$ & $624,83 \pm 83$ & $451,72 \pm 72,31$ & $370,96 \pm 81,87$ & $291,39 \pm 47,75$ & $284,46 \pm 46,64$ \\
\hline Grupo II $(n=11)$ & $997,10 \pm 216,72 *$ & $720,56 \pm 131,96^{*}$ & $551,02 \pm 95,14^{*}$ & $380,01 \pm 102,22$ & $287,36 \pm 35,32$ & $777,35 \pm 274,31^{*}$ \\
\hline Grupo III $(n=4)$ & $815,31 \pm 124,52$ & $670,25 \pm 147,08$ & $563,61 \pm 102,49$ & $431,96 \pm 79,80$ & $375,22 \pm 79,80$ & $851,35 \pm 257,64$ \\
\hline Grupo IV $(n=6)$ & $753,60 \pm 154,67$ & $686,21 \pm 168,68$ & $627,92 \pm 107,36$ & $591,01 \pm 155,86^{*}$ & $511,83 \pm 115,2^{*}$ & $885,49 \pm 401,97$ \\
\hline
\end{tabular}

$\mathrm{x} \pm \mathrm{DE} ;{ }^{*} \mathrm{p}<0,05$ : Valor estadísticamente significativo respecto a su grupo Control
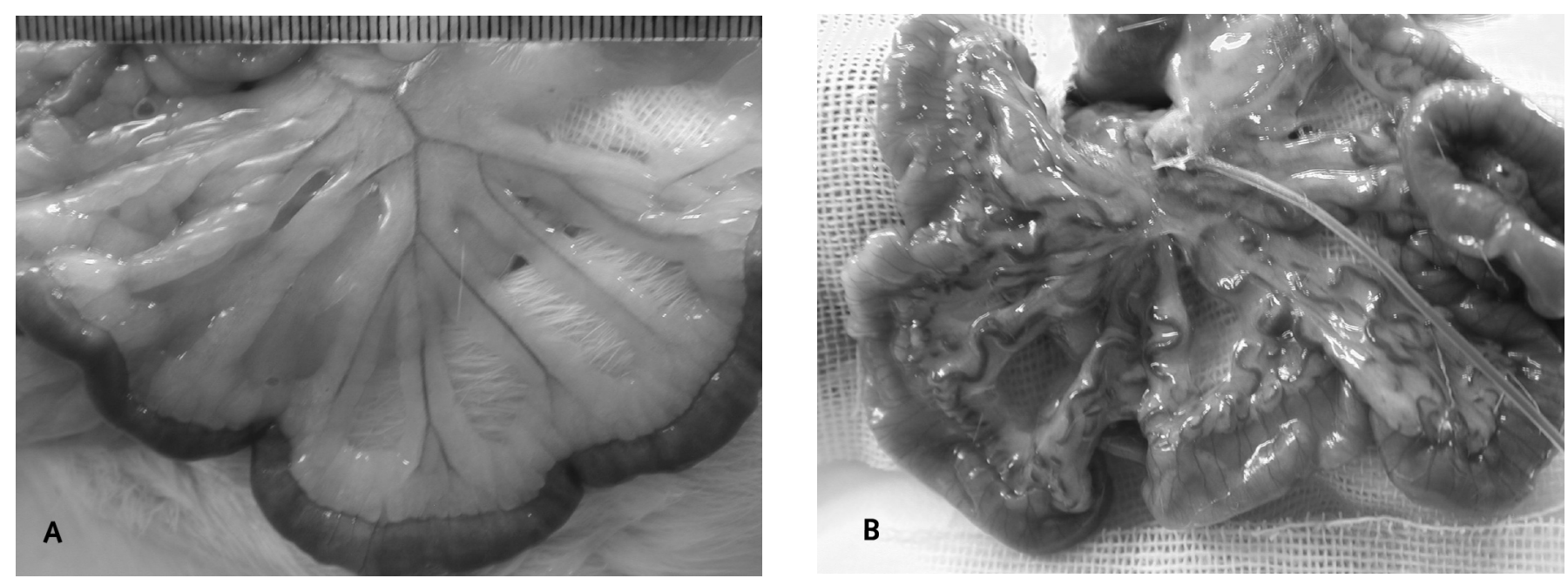

Fig. 1. A: Circulación venosa mesentérica en una rata control de un año de evolución. B: Vasculopatía mesentérica en una rata con hipertensión portal al año de evolución. Se observan la tortuosidad y dilatación de las ramas de la vena mesentérica superior, que es más intensa en las ramas distales (de tercer y cuarto orden).

\section{CÉLULAS CALICIFORMES INTESTINALES}

La cuantificación de células caliciformes en el duodeno de animales con hipertensión portal demuestra que existe un aumento de este tipo de células tanto al mes (grupo II) $(\mathrm{p}<0,01)$ como al año de evolución (grupo IV) $(\mathrm{p}<0,01)$ post-TEVP (Fig. 2).

Este incremento en la densidad de células caliciformes en el duodeno es superior en el grupo IV (TEVP 1 año) respecto del grupo II (TEVP 1mes) (p < 0,01) (Tabla III).

El recuento de células caliciformes en el yeyuno y en el ileon aumenta al mes de evolución en las ratas con hipertensión portal, si bien éstas diferencias no son estadísticamente significativas (Tabla III).

\section{NÚMERO DE MITOSIS EN LAS CRIPTAS}

El número de células en mitosis en las criptas aumentan al año respecto al mes de evolución en los animales control (Tabla IV). En las ratas con hipertensión portal al mes de evolución se produce un descenso del número de células en mitosis en las criptas en el duodeno $(\mathrm{p}<0,01)$ y en el íleon (Tabla
IV). Al año de evolución postoperatoria disminuyen el número de células en mitosis en todo el intestino delgado, si bien éste descenso sólo es estadísticamente significativo en el duodeno $(\mathrm{p}<0,05)$ y en el ileon $(\mathrm{p}<0,01)$ (Tabla IV).

\section{DISCUSIÓN}

Las alteraciones macrovasculares y epiteliales esplácnicas que se producen a largo plazo ( 1 año) en ratas con hipertensión portal prehepática, sugieren la existencia de un proceso de remodelación.

Las alteraciones morfológicas del árbol venoso mesentérico son diferentes en los distintos periodos evolutivos de la hipertensión portal en que han sido estudiadas. A corto plazo (1 mes post-TEVP), el aumento del diámetro de la vena mesentérica superior y de sus ramas de primer y segundo orden reflejan la dilatación proximal que sufre el sistema venoso esplácnico. Este cambio morfológico puede ser secundario a la estenosis de la vena porta, con aumento de la presión portal en la porción proximal del sistema venoso esplácnico que, a su vez favorecería la instauración de la circulación colateral portosistémica, tanto de tipo paraesofágico, como 

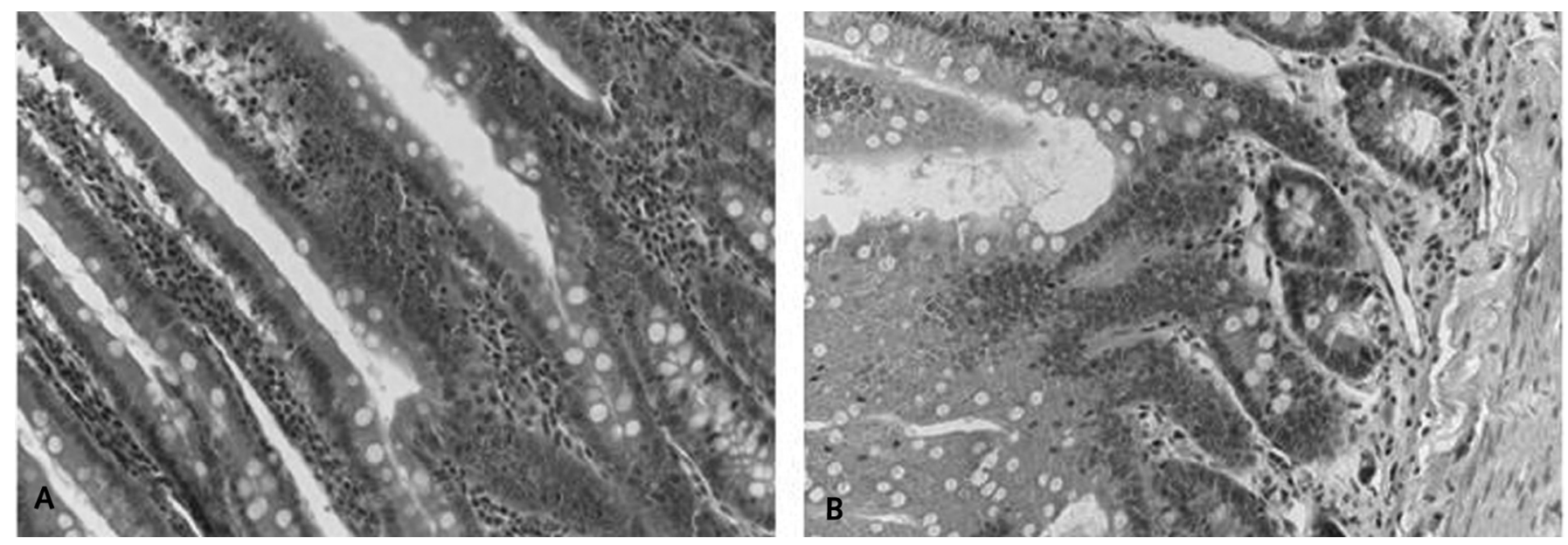

Fig. 2. A. Microfotografía de una sección duodenal de una rata control de un año de evolución. b: Microfotografía de una sección duodenal de una rata con hipertensión portal de un año de evolución, en la cual se observa un aumento de la infiltración de la mucosa por células caliciformes. (H\&E, 20x).

TABLA III

NÚMERO DE CÉLULAS CALICIFORMES/1000 $\mu^{2}$ DE EPITELIO GLANDULAR EN LOS ANIMALES DE LOS GRUPOS I

(CONTROL) Y II ( CON TRIPLE LIGADURA ESTENOSANTE DE LA VENA PORTA) AL MES DE EVOLUCIÓN Y EN LOS GRUPOS III ( CONTROL) Y IV (CON TRIPLE LIGADURA ESTENOSANTE DE LA VENA PORTA) AL AÑO DE EVOLUCIÓN

\begin{tabular}{lccc}
\hline Grupos & Duodeno & Yeyuno & Íleon \\
\hline Crupo I $(n=5)$ & $80,13 \pm 23,67$ & $126,75 \pm 30,68$ & $229,60 \pm 51,63$ \\
Grupo II $(n=10)$ & $120,87 \pm 36,60^{*}$ & $150,51 \pm 25,79$ & $288,32 \pm 102,13$ \\
Grupo III $(n=9)$ & $128,50 \pm 28,02$ & $146,65 \pm 32,91$ & $193,28 \pm 45,50$ \\
Grupo IV $(n=11)$ & $168,37 \pm 41,09 *$ & $132,43 \pm 29,22$ & $195,03 \pm 36,04$ \\
\hline
\end{tabular}

$x \pm D E ;{ }^{*} p<0,05$ : valor estadísticamente significativo respecto a su grupo control.

esplenorrenal, y de la circulación colateral porto-hepática, en particular a través de la vena hepática accesoria (10).

Por el contrario, a largo plazo (1 año post-TEVP) las ratas con hipertensión portal presentan aumento del diámetro de las ramas de tercer y cuarto orden de la vena mesentérica superior, lo que implica la existencia de dilatación distal del sistema venoso esplácnico. Una de las causas posibles de ésta alteración morfológica, sería la persistencia de la circulación hiperdinámica esplácnica que se instaura en estadios evolutivos precoces. El incremento del flujo sanguíneo en el sistema venoso portal se establece por la vasodilatación arteriolar esplácnica que produce circulación hiperdinámica o hiperemia esplácnica (12). La circulación hiperdinámica ha sido atribuida al aumento de sustancias vasodilatadores circulantes y a la disminución de la respuesta a sustancias vasoconstrictoras (13-15). Entre los factores que producen vasodilatación destacan oxido nítrico (NO) (16), monóxido de carbono (CO) (17), factor tumoral de necrosis alfa (TNF- $\alpha$ ) (18) y glucagon (19). A su vez, la hiporeactividad a los vasoconstrictores endógenos, como son noradrenalina, endotelina y vasopresina, refleja la existencia de una respuesta vasoconstrictora anómala o deficiente que, por lo tanto, contribuye a la vasodilatación (20).
TABLA IV

NÚMERO DE CÉLULAS EN MITOSIS/10 CRIPTAS EN INTESTINO DELGADO EN LOS ANIMALES DE LOS GRUPOS I (CONTROL) Y II ( CON TRIPLE LIGADURA ESTENOSANTE DE

LA VENA PORTA) AL MES DE EVOLUCIÓN Y EN LOS GRUPOS III ( CONTROL) Y IV (CON TRIPLE LIGADURA ESTENOSANTE DE LA VENA PORTA) AL AÑO DE EVOLUCIÓN

\begin{tabular}{lccc}
\hline Grupos & Duodeno & Yeyuno & Íleon \\
\hline Grupo I $(n=5)$ & $12,4 \pm 2,3$ & $7,8 \pm 5,9$ & $7,2 \pm 4,4$ \\
Grupo II $(n=10)$ & $7,6 \pm 3,1^{* *}$ & $8,9 \pm 3,3$ & $6,6 \pm 2,8$ \\
Grupo III $(n=9)$ & $19,6 \pm 8,0$ & $15,4 \pm 4,3$ & $15,0 \pm 4,6$ \\
Grupo IV $(n=11)$ & $12,5 \pm 4,9^{*}$ & $11,1 \pm 4,2$ & $7,7 \pm 3,8^{* *}$ \\
\hline
\end{tabular}

$x \pm D E ;{ }^{*} p<0,05,{ }^{*} p<0,01$ : valor estadísticamente significativo respecto a su grupo control.

En las ratas con TEVP de 1 año de evolución persiste la hipertensión portal, si bien es de menor intensidad que en las fases precoces (Tabla I). Sin embargo, el incremento de la presión venosa en el sistema esplácnico podría inducir la acción de fuerzas mecánicas en las venulas postcapilares y, por lo tanto, la correspondiente activación biomecánica del endotelio vascular $(21,22)$. En particular, la estimulación biomecánica de la célula endotelial puede causar su remodelación (22). Asimismo, el estrés mecánico puede inducir la expresión de genes relacionados con la respuesta inflamatoria, esto es, genes que codifican sustancias vasoactivas y factores de crecimiento (21) cuya difusión y acción crónica en la mucosa intestinal implicaría su remodelación.

En esta fase ya crónica de la hipertensión portal experimental también se asocia una disminución de la circulación colateral portosistémcia, así como de la frecuencia e intensidad de la vasculopatía venosa mesentérica, considerada esta como una dilatación y tortuosidad de las ramas de la vena mesentérica, ya espontánea o provocada por la maniobra de Pringle (11).

A su vez, el hígado, atrófico en la hipertensión portal experimental precoz (1 mes post-TEVP), cursa con degeneración grasa en fase crónica (1 año post-TEVP) (23). La esteato- 
sis hepática explicaría porqué no se produce disminución del peso del hígado en las ratas con hipertensión portal crónica (Tabla I).

El incremento de células caliciformes en la mucosa intestinal de las ratas con hipertensión portal es una alteración estructural significativa, máxime al año de evolución. La hiperplasia de células caliciformes con hipersecreción de moco es considerada una alteración propia de la remodelación epitelial del tracto respiratorio en procesos inflamatorios crónicos, como son el asma y la enfermedad pulmonar obstructiva crónica $(5,24,25)$. Este mismo sentido se podría atribuir a la hiperplasia de células caliciformes intestinales en la hipertensión portal prehepática experimental crónica. En éste supuesto, la respuesta hiperplásica de las células caliciformes podría estar mediada por estímulos luminales, esto es, alteraciones de la flora intestinal o por mediadores inflamatorios endógenos (26).

La asociación de hiperplasia de células caliciformes intestinales con la disminución del número de mitosis de las células de las criptas en las ratas con hipertensión portal aguda y crónica sugiere la existencia de una interrelación entre ambos tipos celulares. En éste sentido, el aumento de células caliciformes y la disminución de la proliferación de las células de las criptas supondría una significativa alteración estructural del epitelio intestinal en la enteropatía hipertensiva portal, tanto aguda como crónica.

Se ha sugerido que la hipertensión portal aumenta la susceptibilidad de la mucosa del intestino delgado a la agresión (27). Ya que en las ratas con TEVP a corto plazo se produce un aumento del número de células cebadas en el intestino delgado proximal (4), se podría considerar que éstas células inflamatorias podrían ser responsables del aumento de la susceptibilidad de la mucosa intestinal a estímulos nocivos en la hipertensión portal. En éste caso, la activación de las células cebadas con liberación de sus mediadores podría causar episodios inflamatorios de número e intensidad variables en cada individuo. Asímismo, en fases crónicas (1 año post-TEVP) la hiperplasia de las células caliciformes podría ser también atribuida, entre otros factores, a la persistente infiltración de la mucosa por células cebadas. En éste supuesto, la persistente inflamación de la mucosa intestinal en las ratas con TEVP causaría a largo plazo su remodelación.

En conclusión, las alteraciones morfológicas vasculares y epiteliales que presentan a largo plazo las ratas con hipertensión portal podrían ser consideradas elementos propios de un proceso de remodelación esplácnica y, por lo tanto, sugieren la existencia de una etiopatogenia inflamatoria de la enteropatía hipertensiva portal.

\section{Bibliografía}

1. Viggiano TR, Gostout CJ. Portal hypertensive intestinal vasculopathy: A review of the clinical, endoscopic and histoclinical, endoscopic and histopathologic features. Am J Gastroenterol 1992; 87: 944-954.

2. Sarfeh IJ, TarnawskiA. Gastric mucosal vasculopathy in portal hypertension. Gastroenterology 1987; 93: 1129-1131.

3. Nagral AS, Joshi AS, Bhatia S J, Abraham P, Mistry FP, Vora IM. Congestive jejunopathy in portal hypertension. Gut 1993; 34: 694-697.

4. Díez-Arias J A, Aller MA, Palma MD, Arias JL, Muñiz E, Sánchez M, Arias J. Increased duodenal mucosa infiltration by mast cells in rats with portal hypertension. Dig Surg 2001; 18: 34-40.

5. Davies DE, Wicks J, Powell RM, Puddicombe SM, Holgate ST. Airway remodeling in asthma: New insights. J Allergy Clin Immunol 2003; 111: 215-225.

6. Monterde G, Rodriguez-Fabian G, Vara E, Lopez L, Arias JI, Aller MA Arias J. Increased plasma levels of corticosterone and prolactin and decresed T3 and T4 levels in short-term prehepatic portal hypertension in rats. Dig Dis Sci 2000; 45: 1865-1871.

7. Chojkier M, Groszmann RJ. Measurement of portal-systemic shunting in the rat using g-labeled microspheres. Am J Pathol 1981; 240: G371-G375.

8. Castañeda B, Dubernardi-Venon W, Bandi JC, Andreu V, Pérez del Pulgar S, Moitinho E, Pizcuela P, Bosch J. The role of portal pressure in the severity of bleeding in portal hypertensive rats. Hepatology 2000; 31: 581-586.

9. Kravetz D, Sikuler E, Groszmann RJ. Splanchnic and systemic hemodynamics in portal hypertensive rats during hemorrhage and blood volume restitution. Gastroenterology 1986; 90: 1232-1240.

10. Rodríguez-Fabián G, Monterde G, Diéguez B, Aller MA, Arias J. Hipertension portal a largo plazo en la rata por triple ligadura estenosante de la vena porta. An Med Interna (Madrid) 2000; 17: 137-141.

11. Aller MA, Nava MP, Palma MD, López L, Arias JL, Vara E, Arias J. Redistribución del peso lobular hepático en ratas con hipertensión portal prehepática crónica. Cir Esp 2001; 70: 235-241.

12. Sikuler E, Kravetz D, Groszmann RJ. Evolution of portal hypertension and mechanisms involved in its maintenance in a rat model. Am J Physiol 1985; 248: G618-G625.

13. Macmathuna P, Vlavianos P, Westaby D, Williams R. Pathophysiology of portal hypertension. Dig Dis 1992; 10 (suppl 1): 3-15.

14. Bosch J, Pizcueta P, Feu F, Fernández M, García-Pagán JC. Pathophy- siology of portal hypertension. Gastroenterol Clin North Amer 1992; 21: $1-14$.

15. Orloff MJ. Portal hypertension and portacaval shunt. In: Souba WW, Wilmore DW. Surgical Research.1st ed; London; Academic Press, 2001. p. 637-701.

16. Wiest R, Groszmann RJ. Nitric oxide and portal hypertension: Its role in the regulation of intrahepatic and splanchnic vascular resistance. Semin Liver Dis 1999; 19: 441-446.

17. Fernández M, Lambrecht RW, Bonkovsky HL. Increased heme oxygenase activity in splanchnic organs from portal hypertensive rats: role in modulating mesenteric vascular reactivity. J Hepatol 2001; 34: 812-817.

18. López-Talavera JC, Merrill WW, Groszmann RJ. Tumor necrosis factor-a: A major contributor to the hyperdynamic circulation in prehepatic portal-hypertensive rats. Gastroenterology 1995; 108: 761-767.

19. Pizcueta MP, García-Pagán JC, Fernández M, Casamitjana R, Bosch J, Rodes J. Glucagon hinders the effects of somatostatin on portal hypertension. Gastroenterology 1991; 101: 1710-1715.

20. Bomzon A, Blendis LM. Vascular reactivity in experimental portal hypertension. Am J Physiol 1987; 252: G158-G162.

21. Chien S, Li S, Shyy YJ. Effects of mechanical forces on signal transduction and gene expression in endothelial cells. Hypertension 1998; 31 (part 2): 162-169.

22. García-Cardeña G, Coamander J, Anderson KR, Blackman BR, Gimbrone MA. Biomechanical activation of vascular endothelium as a determinant of its functional phenotype. PNAS 2001; 98: 4478-4485.

23. Alonso MJ, Aller MA, Corcuera MT, Nava MP, Gomez F, Angulo A, Arias J. Progressive hepatocytic fatty infiltration in rats with prehepatic portal hypertension. Hepato-Gastroenterol. (en prensa).

24. Chung KF. Cytokines in chronic obstructive pulmonary disease. Eur Resoir J 2001; 34 (Supl. 1): 50S-59S;

25. Fahy JV. Remodeling of the airway epithelium in asthma. Am J Respir Crit Care Med 2001; 164: S46-S51.

26. De Plancke B, Gaskins HR. Microbial modulation of innate defense: goblet cells and the intestinal mucus layer. Am J Clin Nutr 2001; 73 : S1131-S1141.

27. Jonas G, Erickson RA, Morgan T. Effect of portal hypertension on in vivo bile acid-mediated small intestinal mucosal injury in the rat. Dig Dis Sci 1990; 35: 743-748. 